\title{
Les jeux vidéo compétitifs au prisme des jeux sportifs : du sport au sport électronique
}

\section{Nicolas Besombes}

\section{(2) OpenEdition \\ Journals}

Édition électronique

URL : http://journals.openedition.org/sdj/612

DOI : $10.4000 /$ sdj. 612

ISSN : 2269-2657

Éditeur

Laboratoire EXPERICE - Centre de Recherche Interuniversitaire Expérience Ressources Culturelles Education

\section{Référence électronique}

Nicolas Besombes, «Les jeux vidéo compétitifs au prisme des jeux sportifs : du sport au sport électronique », Sciences du jeu [En ligne], 5 | 2016, mis en ligne le 25 février 2016, consulté le 19 avril 2019. URL : http://journals.openedition.org/sdj/612 ; DOI : 10.4000/sdj.612

Ce document a été généré automatiquement le 19 avril 2019

Tous droits réservés 


\title{
Les jeux vidéo compétitifs au prisme des jeux sportifs : du sport au sport électronique
}

\author{
Nicolas Besombes
}

1 Loin d'être un objet homogène, le jeu vidéo est un phénomène social protéiforme et complexe, qu'il est nécessaire de déconstruire d'un point de vue sociologique (Trémel, 2001). Il se décline en une multitude de supports (bornes d'arcade, consoles de salon, ordinateurs, téléphones portables, tablettes, etc.), de thématiques (jeux de combat, de tir, de stratégie, simulations sportives, etc.), de modes de jeu (seul ou en coopération contre le programme informatique, joueur contre joueur...), qui à leur tour, renvoient à des pratiques, des usages, des discours et des représentations différentes (sociabilité, reconnaissance, compétition...). Depuis la fin des années 1990 et l'apparition des jeux en réseau rendue notamment possible par l'essor d'Internet et la massification des connexions personnelles, l'une de ces pratiques vidéoludiques tend à se spécialiser dans l'organisation d'affrontements codifiés entre joueurs, par écran(s) interposé(s), lors de compétitions nationales et internationales de jeux vidéo. Ce phénomène, que les pratiquants nomment eux-mêmes e-sport pour "sport électronique ", connaît une forte popularité en Asie et notamment en Corée du Sud depuis la fin des années 1990 (Paberz, 2012). Bien qu'elles se soient exportées aux États-Unis et en Europe au tournant des années 2000, cette pratique compétitive du jeu vidéo et sa mise en spectacle sont restées méconnues du grand public et des médias généralistes occidentaux jusqu'au début des années 2010 (Besombes, 2015). Depuis, le jeu vidéo compétitif semble sortir du cercle des initiés pour toucher un public de plus en plus nombreux et diversifié en âge et en sexe, passant entre 2013 et 2015 de 71,5 millions ${ }^{1}$ à 188 millions $^{2}$ de personnes à travers le monde qui regardent et/ou jouent régulièrement ou ponctuellement aux jeux vidéo. 


\section{Jeu vidéo compétitif et jeu sportif, une comparaison complexe}

2 À première vue, les similitudes entre sport électronique et sport moderne sont nombreuses. Un rapide tour d'horizon permet de dégager de multiples ressemblances entre ces deux phénomènes : structuration d'équipes encadrées par des manageurs, des entraîneurs et des préparateurs physiques et mentaux ; répétition et spécialisation des tâches lors d'entraînements périodiques ; technologisation des équipements, matériels et vêtements utilisés dans la pratique; organisation de championnats et de tournois sous forme de ligues ou de tableaux à élimination directe; instauration de calendriers spécifiques répartissant les compétitions tout au long de l'année; mise en place d'un corps arbitral garant de l'application correcte du règlement; mise à jour régulière de classements de joueurs et d'équipes; enregistrement de records; médiatisation des événements de grande envergure à travers la presse et les retransmissions à la télévision ou sur Internet; animation et analyse des rencontres déléguées à des commentateurs; spectacularisation croissante des événements majeurs; starification et sponsorisation des joueurs et équipes victorieuses; mesure et culte de la performance, etc. Les exemples abondent et dénotent une réelle volonté de la part du monde du jeu vidéo compétitif de professionnaliser à la fois sa pratique et ses acteurs (Héas \& Mora, 2003). En empruntant à la forme sportive certaines de ses caractéristiques, le sport électronique cherche à mimer les codes d'une pratique sociale légitimée, espérant ainsi acquérir à son tour la reconnaissance d'une société qui a régulièrement stigmatisé la pratique vidéoludique pour les deux grands maux dont elle serait la cause : addiction et violence.

3 La comparaison apparemment évidente de ces deux entités fait cependant régulièrement débat entre les différents acteurs et amateurs du jeu vidéo compétitif, ainsi qu'auprès de personnes extérieures au phénomène, notamment au sein des forums communautaires et de la presse généraliste. À l'heure où le gouvernement soumet un projet de loi sur les usages numériques dans lequel il questionne le cadre législatif des joueurs professionnels de jeux vidéo ${ }^{3}$, les avis des personnes concernées, qu'ils soient mitigés ou parfois plus tranchés, mettent en lumière l'insuffisance de ces analogies de forme entre sport moderne et jeu vidéo compétitif, pour faire de l'e-sport un sport à part entière.

Dès lors, sur quels critères intrinsèques est-il possible de s'appuyer pour mettre objectivement en regard ces deux pratiques sociales contemporaines? Quels traits distinctifs structurels pertinents peuvent éclairer une étude comparative de ces deux faits sociaux ? Ces deux questions serviront de point de départ à notre réflexion. Pour y répondre, il est nécessaire de dissoudre le vernis sportif dont s'est couvert le jeu vidéo compétitif et de tenter d'en cerner la spécificité et les originalités.

5 Afin de proposer des éléments de réponses, nous allons faire appel à la sociologie du sport, qui ne cesse, depuis la fin des années 1960 en France, de chercher à observer, décrire et classer son objet d'étude, pour mieux le définir et le théoriser. Les travaux sociologiques sur le sport se répartissent selon leurs paradigmes théoriques, leurs méthodes ou leurs thèmes, du récit de la genèse des sports modernes à travers la sociologie historique (Guttmann, 1978 ; Elias et Dunning, 1986) à leur diffusion selon les aires culturelles (Pociello, 1981 ; Parlebas, 1986). 
6 Cette contribution vise donc à questionner la place du jeu vidéo compétitif au sein des jeux sportifs et s'inscrit dans la continuité des travaux de recherche amorcés dix ans auparavant en France par Mora (2003, 2005, 2009), et à l'étranger par Wagner (2006), Jonasson \& Thiborg (2010), Witkowski (2009, 2012), Adamus (2012) et principalement Taylor (2010, 2012), dont l'ouvrage suggère explicitement au champ de la sociologie du sport d'investir le phénomène du sport électronique comme objet de recherche ${ }^{4}$. En nous appuyant sur une définition opérationnelle des jeux sportifs et du sport, nous proposons d'analyser la pratique spécifique du jeu vidéo compétitif en centrant nos observations d'une part sur les comportements moteurs de pratiquants réguliers et occasionnels de jeux vidéo compétitifs et d'autre part sur le dispositif organisationnel des événements esportifs majeurs récents.

\section{Le « sport », un terme ambigu}

7 Le terme "sport» est fréquemment employé de nos jours et sa signification paraît limpide. Pourtant, son utilisation révèle une étonnante confusion, puisqu'il peut à la fois désigner une partie d'échecs, un trajet à vélo pour se rendre sur son lieu de travail, un footing dominical dans le parc le plus proche, une partie de basket-ball entre amis sur un playground, un match de football professionnel, ou une finale olympique d'athlétisme. La définition du sport se présente dès lors comme une question centrale et essentielle lorsqu'il s'agit de catégoriser les activités physiques. Les différentes définitions sociales données sont de rigueurs inégales, parfois contradictoires et souvent imprécises. Il ne semble qu'aucune d'elles ne se soit imposée et que les bornes qui délimitent les activités qui sont du sport de celles qui n'en sont pas varient selon les situations et les pays. Même en consultant l'artisan de la réhabilitation des Jeux Olympiques modernes et de la mondialisation du sport, Pierre de Coubertin, force est de constater notre embarras. Le sport, écrit-il, « est le culte volontaire et habituel de l'exercice musculaire intensif incité par le désir du progrès et ne craignant pas d'aller jusqu'au risque» $(1972, p .7)$. La définition reste vague, comme entourée d'un brouillard de connotations moralisantes et psychologisantes. L'homme qui a pourtant consacré sa vie à l'instauration de puissantes institutions garantes de la légitimité des règles sportives et de leur système compétitif reste muet sur ces éléments. Le terme accepte donc de nombreuses significations, il est polysémique et Parlebas est catégorique : « il [en] devient pansémique » (1999, p. 355).

8 Afin d'éviter les confusions et contre-sens, il convient d'effectuer un bref détour étymologique et historique. Le terme « desport » est apparu en France dès le XIII ${ }^{\mathrm{e}}$ siècle et désigne à cette époque "l'ensemble des moyens grâce auxquels le temps passe agréablement : conversation, distraction, badinage aussi bien que les jeux » (Ulmann, 1977, p. 324). Il s'exporte en Angleterre au XIV siècle (« to sport») et qualifie les jeux, amusements et exercices des classes privilégiées. Les prémisses du sport moderne surgissent finalement en Angleterre au cours du XVIII ${ }^{e}$ siècle et coïncident avec l'apparition du régime parlementaire anglais. Ils représentent les deux faces d'un même processus socio-politique: la pacification progressive des mœurs et l'acceptation du principe d'alternance des vainqueurs tant dans l'hémicycle que dans les stades (Elias \& Dunning, 1986). Dans les deux cas, politique et sportif, il convient de s'entendre sur un système de règles communes, sur la mise en place d'un arbitrage et sur le respect du perdant. Selon Elias et Dunning, l'un des premiers exemples historiques de transformation d'un loisir en sport est la chasse au renard, particulièrement appréciée 
des grands propriétaires anglais de la seconde moitié du XVIII siècle. Cette pratique devient un passe-temps très spécialisé, subordonné à une organisation et à un ensemble de règles bien spécifiques. Non seulement les chasseurs s'interdisent de tuer un autre animal que le renard au cours de ce type de chasse, mais la mise à mort du renard est déléguée à la meute de chiens. Cet ensemble de règles vise alors à accroître la difficulté de la chasse, à prolonger la traque et à retarder l'instant de la mise à mort. De fait, le plaisir que procure l'activité réside bien plus dans la poursuite de la proie que dans son exécution. L'entreprise devient désintéressée : le gibier abattu ne sera pas consommé, la chasse valant pour elle-même et s'avérant être tout autant un agent de régulation sociale que de pacification des mœurs. Lorsque le sport se propage finalement en Europe au XIX siècle, le terme est chargé de sa signification britannique et renvoie aux manières hypercodifiées de se divertir de la noblesse et de la bourgeoisie. Le sport est fondamentalement le reflet de la culture qui l'a vu naître, une création sociale, historiquement datée et située (Ulmann, 1977 ; Elias \& Dunning, 1986 ; Parlebas, 1986 ; Defrance, 1995).

9 L'indéniable complexité à définir le phénomène sportif n'a cependant pas découragé certains auteurs en sciences sociales de tenter de dégager les traits différentiels intrinsèques qui distinguent les pratiques sportives des autres pratiques sociales. En France, à compter des années 1960-1970, les définitions des experts en sciences humaines et sociales prévalent sur celles des acteurs qui instituent les activités physiques et sportives (Defrance, 1995). Les premiers travaux définissent le sport comme une activité contextuelle, et révisable au cours du temps, et insistent sur les traits originaux du sport par rapport aux jeux physiques des civilisations antérieures que sont les agôns de la Grèce antique (Jeux Pythiques, Isthmiques, Néméens et les plus illustres de tous, Olympiques), les ludi de l'Empire romain (jeux de la gladiature et courses de chars) et les jocus médiévaux (joutes et tournois chevaleresques). En effet, bien que Grecs et Romains aient développé des activités corporelles dont les formes gestuelles et règlementaires pourraient suggérer une continuité avec les pratiques sportives contemporaines, telles que la lutte, la course à pied ou le lancer du disque, il s'avère que les Jeux de l'Antiquité relèvent fondamentalement de registres religieux et sacrés, et ne peuvent de ce fait, être assimilés aux sports modernes. Quant aux jeux et exercices du Moyen-âge et de la Renaissance, leur signification socioculturelle ne peut être associée aux rencontres sportives modernes, leur enjeu étant bien souvent l'identité d'une communauté ou d'une localité, servant parfois même de rite d'intégration, notamment pour les jeunes hommes célibataires (Terret, 2007). Pour construire une définition, les auteurs en sciences sociales étudient les sports dans leurs relations concrètes avec les multiples institutions sociales, écartant ainsi la tentation de caractériser le sport par quelque vertu. Ainsi en est-il de Magnane (1964) pour qui le sport est « une activité de loisir dont la dominante est l'effort physique, participant à la fois du jeu et du travail, pratiquée de façon compétitive, comportant des règlements et des institutions spécifiques, et susceptible de se transformer en activité professionnelle» $(1964$, p. 81$)$. Allant dans ce sens, Parlebas distingue quant à lui, les « jeux sportifs » de leur forme institutionnelle, le «sport ». Le jeu sportif, écrit-il, est une «situation motrice d'affrontement codifiée, dénommée "jeu" ou "sport" par les instances sociales" (1999, p.196). Il identifie et isole ainsi quatre critères objectifs et nécessaires à la définition du sport. Selon lui, l'activité est de nature motrice, elle est règlementée, donne lieu à la compétition et se manifeste par une prise en compte institutionnelle. À l'instar de Magnane (1964), Callède (1993) et Defrance (1995), Parlebas (1999) distingue, à l'aide des critères institutionnel, compétitif et règlementaire, les activités physiques libres et informelles telles que la baignade ou le jogging, des sports 
fédéraux que sont la natation ou le demi-fond en athlétisme. Toutefois, dans sa définition, Parlebas substitue le critère de l'effort physique au profit de celui de " pertinence motrice ${ }^{5}$ ». Cette caractéristique permet de prendre en compte dans les sports, des activités telles que le tir à l'arc, le tir au pistolet, le curling, la pétanque ou le golf, dont la dépense énergétique relative semble moindre.

L'objectif de cet article est donc de confronter, l'un après l'autre, les quatre critères de définition que nous avons retenus (pertinence motrice, compétition, règlementation et institutionnalisation) à la pratique compétitive des jeux vidéo à travers l'exemple de l'un des jeux les plus médiatisés de ces trois dernières années : League of Legends, développé et édité par Riot Games depuis 2009. Cet arène de bataille en ligne multijoueur (ou MOBA en anglais pour Multiplayer Online Battle Arena) en jeu en ligne gratuit (Free-to-play en anglais, jeu dans lequel il est tout de même possible d'acheter des biens virtuels), rassemble aujourd'hui près de 67 millions de joueurs à travers le monde chaque mois ( 27 millions chaque jour et plus de 7 millions simultanément) ${ }^{6}$. Il se pratique de manière compétitive par équipes de cinq joueurs lors d'événements pouvant être parfois suivis par plus de 30 millions de viewers (internautes-téléspectateurs) et lors desquels les vainqueurs peuvent remporter des sommes atteignant le million de dollars. Ce jeu, à l'instar de quelques autres titres (Starcraft II, 2010, développé et édité par Blizzard Entertainment ou CounterStrike Global Offensive, 2012, développé et édité par Valve Corporation), est régulièrement utilisé par les médias généralistes (télévision, radio ou presse écrite) comme vitrine du mouvement e-sportif.

\section{Le jeu vidéo compétitif, une pratique de nature motrice}

11 Le discours commun a tendance à opposer jeux vidéo et activités physiques et sportives. Là où le sport serait notamment synonyme de dépense énergétique et d'activité de plein air, le jeu vidéo serait, quant à lui, porteur de la représentation opposée : activité d'intérieur, peu propice à l'effort physique. Par un effet de glissement sémantique, les qualités attribuées aux pratiques se sont progressivement appliquées aux pratiquants: c'est ainsi que les uns seraient actifs et en pleine possession de leurs moyens physiques, tandis que les autres seraient in-actifs, et subordonnés à la machine (Peter, 2007). La place du corps émerge donc d'abord de façon négative dans les imaginaires liés aux jeux vidéo, cette représentation prenant principalement racine dans l'avènement de l'ère industrielle, technique puis technologique, qui réduirait, voire annihilerait la place du corps au profit de la machine (Roustan, 2004).

12 Pourtant, le concept d' "Homme total» de Mauss (1950) permet d'entrevoir les mécanismes de mise en jeu du corps dans la pratique vidéoludique, tout particulièrement à travers la place centrale qu'occupent à la fois la dextérité digitale (des doigts) du joueur, ses compétences cognitives et sa faculté à contrôler ses émotions dans l'action du jeu. En effet, comme le suggère Roustan (2003), le joueur met en œuvre toute la complexité de ses dimensions biologiques, psychologiques et sociales lorsqu'il joue, engageant son corps tout entier dans la pratique du jeu vidéo. Il est stimulé au niveau de son attention, de ses perceptions, de ses émotions et de ses sensations. La totalité de son corps est dans l'action, en contact avec les objets matériels qui le soutiennent (écran, clavier, souris, manette, fauteuil) et les personnes qui peuvent l'entourer. Les perceptions du jeu, visuelles via l'écran, tactiles via les manettes vibrantes par exemple et auditives via les périphériques audio (principalement le casque), donnent au joueur des sensations 
auxquelles il répond par des comportements moteurs sur les périphériques de contrôle : pression sur les touches de la manette ou du clavier et/ou déplacement de la souris. Le joueur fait alors corps avec les différents éléments sensori-affectivo-moteurs de son environnement, et finit par s'identifier totalement aux mouvements du personnage virtuel qu'il incarne à l'écran (Clais et Roustan, 2003). Il n'a plus l'impression de contrôler ses doigts, mais bien le personnage virtuel lui-même : lorsqu'il fait courir l'avatar qu'il contrôle à l'écran, il ne pense pas à appuyer sur les touches le lui permettant, il court. Cette projection des sensations du joueur dans l'univers virtuel peut s'expliquer par ce que Jean-Pierre Warnier (1999) nomme l'incorporation de la dynamique des objets. Le corps perceptif du joueur s'étend jusque dans le clavier et la souris, avec lesquels il développe des automatismes moteurs, "qui ont pour résultat une grande économie d'énergie et une capacité à agir longtemps sans fatigue » (Warnier, 1999, p. 12). À partir d'un certain niveau d'expertise, grandement lié à l'apprentissage par répétition, le niveau de conscience réflexive diminue et le joueur oublie ses doigts pour déporter ses sensations et son attention sur le personnage virtuel qu'il incarne. Clavier, souris, manettes ou joysticks apparaissent comme des « orthèses » sensorielles, non pas du corps organique du joueur, mais de son corps perceptif et de son schéma corporel (voir Figure $1)^{7}$.

Figure 1 : Le corps comme support de la pratique vidéoludique

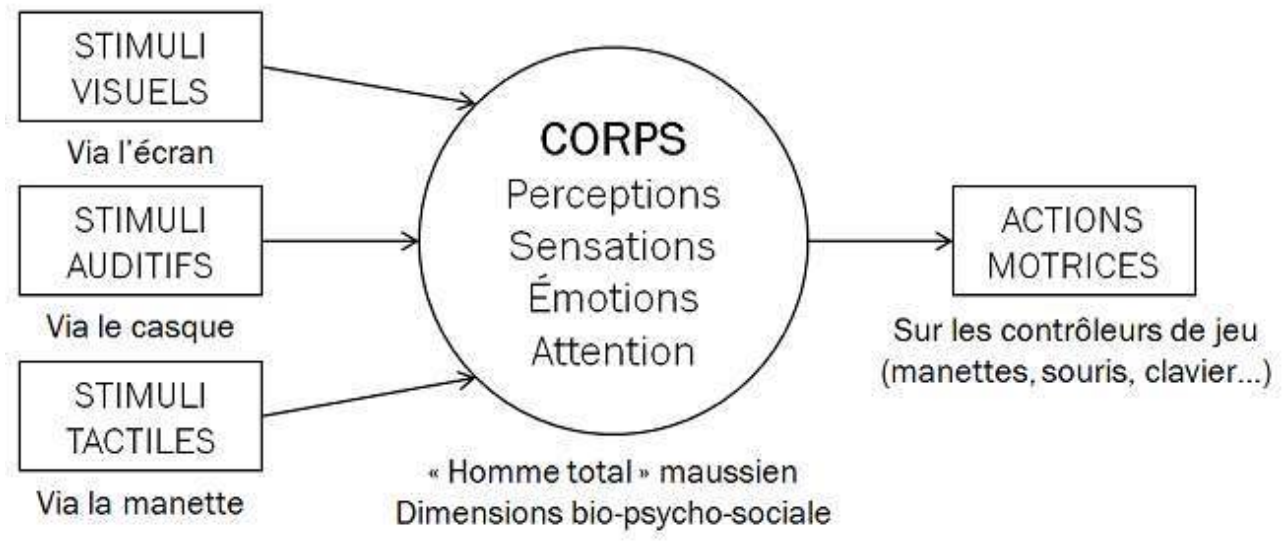

13 Dès lors, comme le suggèrent Clais et Roustan (2003), incorporer la dynamique d'un jeu vidéo exige tout d'abord d'incorporer la dynamique du matériel informatique utilisé. Le cas de League of Legends, qui se joue sur ordinateur, nécessite dans un premier temps de la part du joueur d'appréhender les caractéristiques physiques du clavier et de la souris, c'est-à-dire de savoir où se situent les touches les plus utilisées ainsi que de manipuler la souris de manière efficace. En effet, pratiquer en regardant les touches du clavier risque de faire perdre au joueur le fil de la partie dans laquelle il est engagé. Cette "prise en main », ou "engagement haptique » pour reprendre le terme de Witkowski (2012), s'obtient assez rapidement au fil de l'expérience ou s'acquiert délibérément par l'entraînement, afin d' "apprendre aux doigts " les gestes à automatiser jusqu'à l'inconscient moteur ${ }^{8}$ (le stade du réflexe). Cette appropriation du matériel s'oublie relativement vite mais peut ressurgir lors de changements de supports et d'interface de contrôle (d'un ordinateur vers une console et inversement, ou bien d'une console à une autre, et donc d'une manette à une autre), obligeant le joueur à reprendre conscience de ses actions. 

(intitulé des modes d'emploi affichés sur les bornes d'arcade anglo-saxonnes, abréviation de " how the game plays »). Le gameplay désigne la façon dont le jeu se joue : telle touche ou combinaison de touches sur le périphérique de contrôle permet au personnage virtuel d'effectuer telle action à l'écran. Tout jeu vidéo se définit par un répertoire d'actions offertes au joueur, qui peuvent varier en qualité et en quantité. Il sera ainsi question d'un gameplay pauvre si les actions possibles sont peu nombreuses ou peu variées, et, à l'inverse, d'un gameplay riche si les actions possibles permettent un grand nombre de combinaisons et encouragent des interactions plus variées avec l'environnement virtuel. Le jeu League of Legends nécessite ainsi l'automatisation d'actions plus ou moins complexes et rythmées sur les touches du clavier, ainsi que l'optimisation de la rapidité et de la précision du geste de la main commandant le mouvement de la souris. Les touches $\mathrm{A}, \mathrm{Z}, \mathrm{E}$, $\mathrm{R}, \mathrm{S}$ et $\mathrm{D}$ du clavier permettent ainsi de déclencher les différentes capacités du personnage (quatre « compétences » uniques et deux «sorts d'invocateurs »), tandis que le clic droit de la souris permet de déplacer le personnage sur le champ de bataille virtuel et le clic gauche d'activer certains effets et bonus particuliers issus des nombreux objets. personnages à incarner dans le jeu, différentes factions à jouer ou plusieurs bolides à piloter, ayant chacun leurs propres caractéristiques et particularités. En janvier 2016, le jeu League of Legends comporte ainsi 128 personnages virtuels, appelés «champions». Chaque champion offre un style de jeu différent, comme le spécialiste du soutien ("support»), celui infligeant des dégâts élevés en une courte période de temps («assassin ») ou encore celui subissant les dégâts à la place de son équipe («tank »). Le joueur doit ainsi incorporer la dynamique de chacun de ces différents personnages et de ses capacités, afin d'en maîtriser les «mécaniques». Les mécaniques de jeu correspondent à la capacité du joueur à effectuer des actions dans le jeu en utilisant les propriétés de son champion de manière optimale, et peuvent s'apparenter à l'aspect technique de la pratique e-sportive. Cela consiste à connaître les nombreuses possibilités du personnage : caractéristiques des quatre différentes « compétences» dont il dispose ainsi que de son "passif », distances et zones d'action précises des animations des sorts sur l'écran, et synchronisations (timings) des combinaisons de touches possibles. Ces 128 personnages virtuels sont donc autant de gameplays différents à incorporer, et révèlent ainsi l'abondance de possibilités de conduites motrices à actualiser de la part du joueur sur les périphériques de contrôle.

16 Le joueur utilise ses mains pour que sa représentation numérique à l'écran puisse interagir avec les autres personnages (se battre contre les adversaires ou protéger ses partenaires) ou avec l'environnement virtuel dans lequel il évolue (se déplacer en évitant les obstacles). Il doit constamment prélever de l'information sur le terrain de jeu virtuel, donner du sens aux animations des personnages contrôlés par les autres joueurs visibles à l'écran, et y répondre par des actions réelles sur le clavier et la souris. Cette aptitude repose en grande partie sur la rapidité : rapidité de décodage de ces animations à l'écran, de traitement des informations pertinentes, de prise de décision, et enfin d'exécution. À la rapidité vient s'ajouter la capacité à réaliser plusieurs tâches en même temps. Les conduites motrices réelles du joueur sur le clavier vont grandement déterminer le déroulement des comportements virtuels exécutés par le personnage qu'il manipule à l'écran : une combinaison de touches mal rythmée, incorrecte ou ratée (ce que les joueurs nomment «missclick», c'est-à-dire une pression du doigt sur une mauvaise touche du 
périphérique de contrôle, ou un clic à un mauvais endroit de l'écran et qui correspondrait, en sport, à un geste technique manqué), ou un déplacement de la souris imprécis, et la partie peut être perdue. Il y a une correspondance directe entre l'action sur le jeu en tant que machine (le clavier et la souris) et l'action sur le jeu en tant que monde virtuel (les images en mouvement à l'écran) (Roustan, 2003). La motricité est à la fois le support et la finalité de la pratique du jeu vidéo compétitif, comme l'atteste la place centrale accordée au perfectionnement de la dextérité du joueur. Les conduites motrices digitales des joueurs sont en effet inlassablement répétées et perfectionnées lors d'entraînements quotidiens, elles sont supervisées par des entraîneurs, favorisées par les évolutions ergonomiques dont les claviers et souris de compétition font l'objet, mesurées à l'aide de statistiques, et constamment analysées par les joueurs eux-mêmes. À l'instar de toute pratique sportive, il est indispensable pour le joueur de League of Legends de maitriser ses conduites motrices digitales, bien que médiées par la machine et le programme informatique, pour espérer pouvoir l'emporter sur ses adversaires.

\section{L'e-sport, un système compétitif qui s'est peu à peu organisé...}

La compétition motrice est une situation objective d'affrontement au cours de laquelle plusieurs individus accomplissent une tâche motrice (Parlebas, 1999). Les formes de compétitions peuvent être très riches en possibles (tous contre un, chacun pour soi, plusieurs équipes les unes contre les autres, etc.).

Dès ses balbutiements dans les laboratoires du Massachussetts Institute of Technology et la création par trois étudiants de SpaceWar! en 1962, le jeu vidéo offre aux joueurs la possibilité de se confronter entre eux par le biais du programme informatique. Historiquement, les premières traces de rencontres compétitives entre joueurs remontent à octobre 1972 et les Intergalactic SpaceWar Olympics à Stanford, dont le magazine Rolling Stone ( $\left.\mathrm{n}^{\circ} 123\right)$ de l'époque relate l'événement. À cette période, le jeu vidéo connaît un succès phénoménal, notamment grâce à la sortie quelques mois plus tôt du jeu Pong, dont la borne d'arcade envahit littéralement les centres commerciaux des ÉtatsUnis. Parallèlement aux premières compétitions de jeux vidéo, avec les jeux d'arcade apparait le principe du meilleur résultat (highscore). Le joueur affronte alors les autres adversaires de manière différée, en tentant de réaliser le score le plus élévé possible. Les performances enregistrées dans le programme informatique servent alors de valeur de référence aux prochains joueurs qui tenteront de les défier. Jusqu'au début des années 1980, d'autres compétitions organisées par le développeur Atari voient épisodiquement le jour sur des jeux tels que Space Invaders (Taito, 1981) ou Pac-Man (Namco, 1980), et réunissent plusieurs milliers de participants. Les années qui suivent sont marquées par la faillite de l'industrie vidéoludique et le désintérêt du public pour les jeux vidéo, en raison d'un trop grand nombre de productions identiques et d'une expérience de jeu inadaptée aux consoles de salon (Triclot, 2011). Cependant, en 1983, l'apparition de Mario Bros. sur les consoles de salon réinvente l'expérience vidéoludique et relance l'économie du marché du jeu vidéo et la production de nouveaux titres. Le début des années 1990 est alors marqué par la suprématie de Nintendo. La firme japonaise organise dans vingt-neuf villes à travers les États-Unis, les Nintendo World Championships en 1990, puis la Nintendo PowerFest en 1994. Lors de ces événements, les compétiteurs, répartis par classes d'âges 
(-11 ans, 12-17 ans, +18 ans), s'affrontent pendant un temps limité sur trois des jeux de la firme, dont on additionne les résultats.

Avec l'apparition d'Internet et le développement des connexions personnelles, la fin des années 1990 marque un tournant dans le jeu vidéo compétitif. Le jeu en réseau permet au pratiquant d'affronter, à n'importe quel moment, des adversaires situés à des milliers de kilomètres et popularise ainsi la compétition entre joueurs. En octobre 1997, la Cyberathlete Professional League (CPL) réunit environ 300 participants et marque le début d'une nouvelle ère pour l'e-sport ${ }^{9}$. La même année, l'Electronic Sports League (ESL) est fondée en Allemagne et organise des compétitions opposant les meilleurs joueurs européens. En octobre 2000, La Corée du Sud inaugure les premiers World Cyber Games (WCG), présentés comme les «Jeux Olympiques du jeu vidéo », tandis que depuis juillet 2003 s'organise en France puis à l'étranger la Coupe du monde des jeux vidéo ${ }^{10}$, dénommée Electronic Sport World Cup (ESWC). Depuis les années 2000, le nombre de compétitions connaît alors une progression fulgurante, passant d'une dizaine de tournois organisés par an en 2000 à près de sept cents en $2010^{11}$. Dernièrement, de nouveaux circuits compétitifs sont venus s'ajouter aux précédents à travers le monde, par exemple la Global Starcraft II League (GSL) depuis août 2010 en Corée du Sud ou encore la North American Star League (NASL) en février 2011 aux États-Unis, témoignant d'une structuration de plus en plus importante du milieu e-sportif au niveau mondial. Ce début de décennie voit alors s'instituer un véritable processus de professionnalisation dans l'organisation des compétitions, les moyens financiers et techniques déployés se rapprochant de plus en plus des standards actuels des spectacles sportifs télévisés.

Aujourd'hui, la mise en place d'un calendrier spécifique pour chaque jeu vidéo compétitif répartit les compétitions tout au long de l'année et rythme les saisons du sport électronique. La périodicité de ces saisons est fournie par la distribution des nombreux événements e-sportifs à travers les mois, et coïncide généralement avec la mise à jour et le rééquilibrage annuel des règles du programme informatique. Ces cycles réguliers conditionnent les temps forts (événements qualificatifs majeurs) et les temps morts (présaison) de l'année, en vue d'engendrer une spectacularité croissante jusqu'à la fin de la saison. Dans le cas du jeu League of Legends, la durée d'une saison est d'environ neuf mois (de fin janvier à fin octobre) durant lesquels les équipes de joueurs professionnels se confrontent lors de différents championnats et tournois qualificatifs. Les événements majeurs, les League of Legends Championship Series (LCS), se tiennent quant à eux pendant 12 semaines, au début du mois de février (le Spring Split) et à la fin du mois d'août (le Summer Split) en vue d'atteindre le point culminant de la fin de saison en octobre: les World Championships Series (WCS) ou phases finales des championnats du monde. Le jeu emprunte au modèle sportif son organisation compétitive en associant à la fois un système de classements pour les affrontements en ligne, qui promeut pendant la saison les meilleurs joueurs ou équipes dans les divisions supérieures, avec un système de championnat qualificatif par étapes successives pour les équipes professionnelles. Les matchs peuvent alors être découpés en manches (une, trois ou cinq), retardant la désignation du vainqueur, et permettant de susciter suspense et émotions chez les spectateurs. Les phases finales des World Championships de 2015, à l'instar de la Coupe du Monde de football FIFA, ont ainsi vu les meilleures équipes professionnelles de cinq zones géographiques (Europe, Amérique du Nord, Corée du Sud, Chine et Taïwan/Hong Kong/ Macao) s'affronter lors d'une première phase de poule en matchs aller-retour, à l'issue de laquelle les deux meilleures équipes de chacune des quatre poules se sont qualifiées pour 
un tableau final à élimination directe en trois matchs gagnants. Les spectateurs (viewers) ont ainsi suivi 73 rencontres en un mois de compétition, avant de connaître l'équipe victorieuse. Aujourd'hui, le sport électronique est donc délibérément orienté vers une organisation compétitive rationnelle de ses événements, favorisant ainsi la spectacularité de sa pratique (voir Figure 2).

Figure 2 : Un système compétitif e-sportif structuré

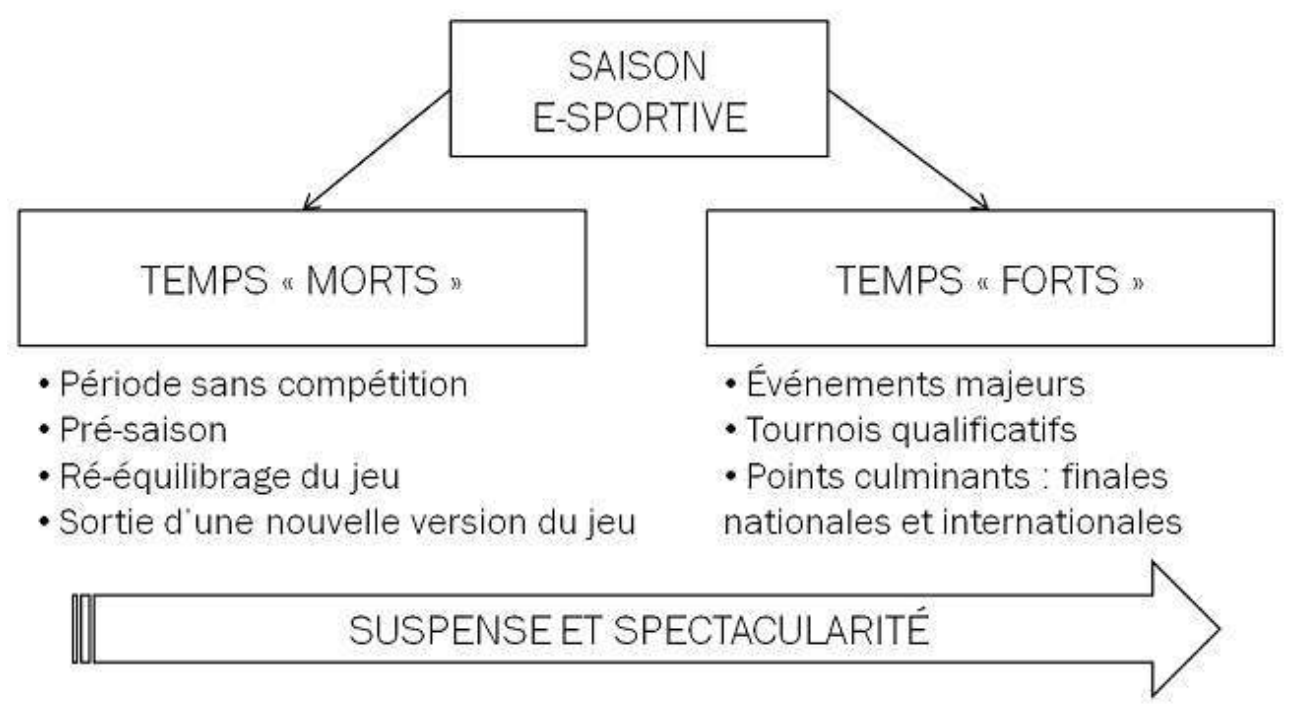

\section{... et réglementé}

La compétition est impérativement soumise à des règles qui en définissent les contraintes, le fonctionnement et tout particulièrement les critères de réussite ou d'échec. En l'absence de règlementation, la pratique sera qualifiée de libre ou d'informelle.

La pratique du sport électronique redonne une place centrale à la règle du jeu, là où le jeu vidéo consacre le programme informatique comme seul arbitre de ce qu'il est possible de faire ou non dans l'univers virtuel (Boutet, 2003). De manière schématique, lorsque le joueur affronte la machine, tout ce que le programme accepte est autorisé. Les garants de ce règlement implicite du logiciel sont les développeurs du jeu et principalement leurs équipes d'équilibrage, qui ont pour responsabilité de faire en sorte que les possibilités dans le jeu n'avantagent ou ne désavantagent pas les joueurs. Cet équilibrage régulier du jeu peut à la fois concerner les caractéristiques du terrain de l'affrontement virtuel (la carte sur laquelle se disputent les rencontres), les propriétés des personnages virtuels (capacités et statistiques) et les équipements et items dans le jeu. Depuis sa sortie officielle en octobre 2009, le jeu League of Legends a ainsi connu près de cent quarante patchs, permettant la mise à jour du logiciel et de ses règles, servant à corriger certains défauts et déséquilibres, et à ajouter de nouvelles fonctionnalités (notamment de nouveaux « champions »).

Cependant, avec le jeu vidéo compétitif, le joueur n'affronte plus seulement le logiciel, mais des adversaires humains dotés d'intentions, de projets, de créativité et de ruse. La machine devient alors moyen et lieu de l'affrontement, tandis que le contrôle de 
l'arbitrage assuré par le programme informatique n'est plus suffisant (Boutet, 2003). À l'instar de la pratique de la chasse au renard de la fin du XVIII ${ }^{\mathrm{e}}$ siècle (Elias et Dunning, 1986), et de tous les sports qui lui succèdent depuis deux siècles, il est nécessaire pour les acteurs du jeu vidéo compétitif d'établir un système de règles, fournissant un cadre de référence identique pour tous les joueurs, et d'en assurer le respect afin d'éviter les phénomènes de triche. L'adoption de ce système de contraintes et de prescriptions précises empêche alors les comportements de joueurs contraires à « l'esprit du jeu ", telle que l'exploitation d'une faille de la programmation («exploiting») ou l'installation d'un programme additionnel permettant de faire ce qui est interdit aux autres dans le monde virtuel («hacking»). Ainsi, le règlement des phases finales des World Championships de League of Legends d'octobre 2015 disputées en Europe (Paris, Londres, Bruxelles et Berlin) comprend 34 pages, et fait preuve d'une certaine exhaustivité montrant une réelle volonté de la part des organisateurs de définir l'univers d'actions dans lequel les joueurs vont pouvoir et devoir évoluer pendant le temps de la compétition ${ }^{12}$. Les règles mentionnent entre autres les conditions de participation et d'éligibilité, les équipements et le matériel technique autorisés pendant la compétition (claviers, souris, casques, tenues), le format des rencontres, les conditions de victoire, le comportement de joueurs proscrits dans le jeu et hors du jeu, le rôle des arbitres chargés de faire appliquer le règlement et le répertoire des sanctions prévues en cas de manquement à l'une de ces prescriptions, de l'avertissement verbal à la disqualification. Le règlement de ces World Championships correspond à un pacte d'association explicite et formel, imposant les modalités particulières d'interactions et de comportements autorisés durant la compétition. Il propose aux joueurs la garantie du respect de ces règles par la codification draconienne de l'espace et de la durée des affrontements, et par le contrôle constant du matériel informatique par l'instance organisatrice de l'événement. Cette règlementation de la compétition engage alors le joueur dans une démarche rationnelle de compréhension de sa pratique et lui assure de disposer des mêmes chances que ses adversaires (voir Figure 3).

Figure 3 : Une pratique compétitive doublement réglementée

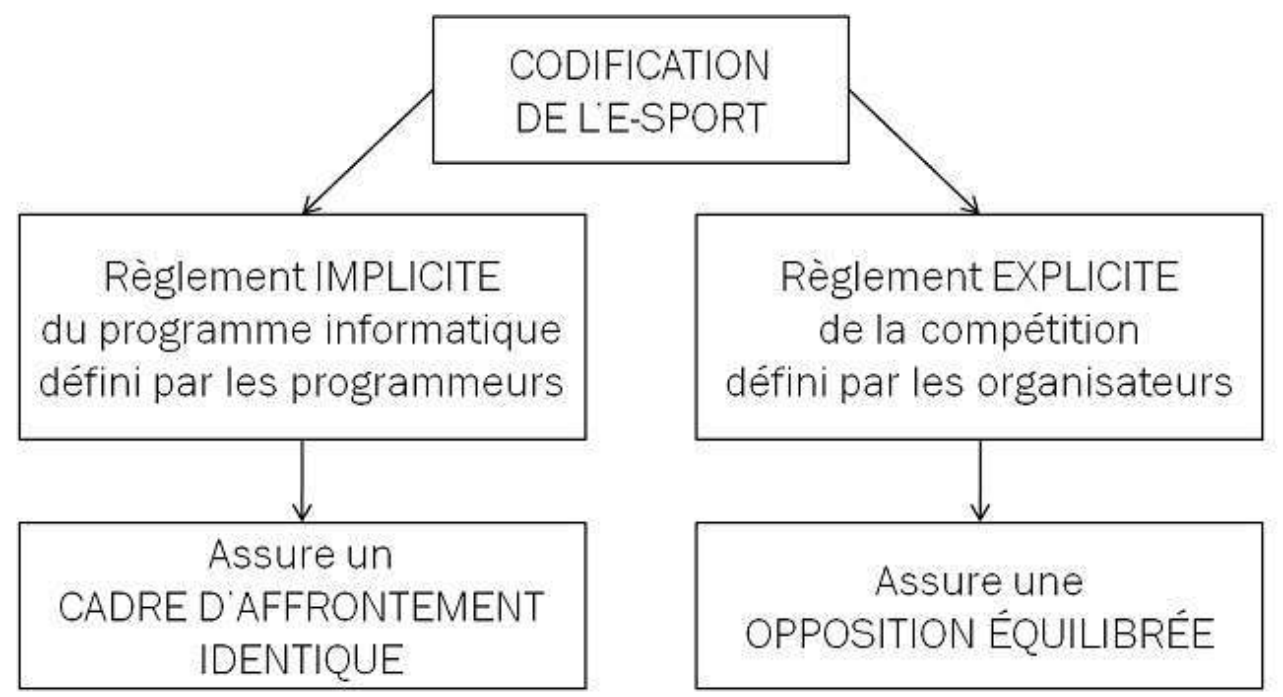

La codification du sport électronique est donc définie d'un côté par ce que le programme informatique régulièrement mis à jour par les développeurs autorise le joueur à faire 
dans l'univers virtuel, et de l'autre par la mise en place par les organisateurs de compétitions d'un système de règles prescrivant les contraintes et possibilités des joueurs autour $\mathrm{du}$ jeu. Là où le programme informatique fournit un cadre implicite d'affrontement identique pour tous les joueurs de toutes les cultures à travers le monde et dont il est le seul arbitre, le règlement e-sportif d'une compétition correspond au pacte explicite, provisoire et restreint d'un événement, qui assure une opposition équilibrée entre tous les participants.

\section{L'émiettement institutionnel e-sportif en France}

Parmi l'ensemble des pratiques ludocorporelles, certaines reçoivent la reconnaissance officielle d'instances sociales et sont gouvernées par des fédérations de tutelle représentatives du pouvoir étatique d'une part (les fédérations nationales et le comité national olympique), et par des organisations internationales spécifiques d'autre part (les fédérations internationales et le comité international olympique). Or, si le jeu vidéo compétitif est bien une pratique de pertinence motrice, structurée sous forme de compétitions, et règlementée par un contrat formel, il s'affranchit clairement du critère institutionnel fédéral classique et s'éloigne ainsi du sport moderne. En France, malgré de nombreuses tentatives, dont la dernière officielle date de fin novembre 2015 à la suite de "l'eSport Summit »", les acteurs du sport électronique n'ont jamais réussi à faire reconnaitre leurs instances dirigeantes par le ministère des Sports. Depuis la Fédération Française de Jeux en Réseaux (FFJR), en passant par celle de Cyber-Sport (FFCS) créée en 2002, celle des Jeux Multi Joueurs (FFJMJ) déclarée en 2005, celle de Jeux Vidéo en Réseau (FFJVR) qui semblait réussir à fidéliser un peu plus de 2000 joueurs depuis 2004, ou bien encore la sobrement intitulée Fédération Française de Jeu Vidéo (FFJV) qui promouvait depuis 2013 la pratique compétitive de jeux de simulation sportive (le football avec FIFA [Electronic Arts, à partir de 1993] et la course automobile avec Gran Turismo [Polyphonie digital, à partir de 1997] ou TrackMania [Nadeo, à partir de 2003]), toutes ces associations organisatrices d'événements e-sportifs qui avaient pour ambition de légitimer la pratique du jeu vidéo compétitif à un niveau fédéral, ont fini dans le meilleur des cas par s'affaiblir, et dans le pire par s'éteindre (Mora, 2005). Aujourd'hui, seules les associations apportant une expertise organisationnelle, logistique et humaine de longue date dans le monde vidéoludique, ainsi que de nouvelles ressources matérielles et économiques tout en proposant parfois un accompagnement spécifique aux joueurs amateurs et semiprofessionnels, semblent réussir à structurer et fidéliser la communauté e-sportive de manière durable. Il en est ainsi par exemple des Masters Français du Jeu Vidéo (MFJV) depuis 2009, ou du Stunfest depuis 2005. Les MFJV, organisés et supervisés par l'association LAN Alliance, sont actuellement l'un des circuits de jeux vidéo compétitifs sur ordinateurs et consoles les plus attractifs et reconnus en France, avec un peu moins de trente étapes dispensées de septembre 2014 à juin 2015, dont la Gamers Assembly (GA) à Poitiers et la Lyon e-sport, qui sont deux des événements les plus importants en nombre de participants, de jeux représentés et de visiteurs (environ 1600 compétiteurs et près de 15000 visiteurs lors des quatre journées de la GA 2015). Chaque saison se conclut par des phases finales qui récompensent les meilleurs joueurs et équipes, en les invitant à s'affronter dans un ultime tournoi lors duquel les vainqueurs de chaque jeu se verront décerner le titre de «champion de France ». Le Stunfest, quant à lui, est une compétition internationale de jeux vidéo de combat sur console (Versus Fighting) organisée par 
l'association 3 Hit Combo, et qui se déroule chaque printemps à Rennes. L'édition de 2015 a ainsi vu plus de 800 joueurs venus du monde entier (Europe, Asie et Amérique du Nord) s'affronter devant 11000 visiteurs trois jours durant lors d'une quinzaine de tournois.

Si l'organisation des saisons et des compétitions e-sportives françaises est structurée par de nombreux acteurs associatifs qualifiés et expérimentés, il n'en reste pas moins que l'institutionnalisation fédérale $\mathrm{du}$ sport électronique en France s'avère totalement inexistante puisqu'illégitime aux yeux du ministère de la Ville, de la Jeunesse et des Sports. Cette structuration associative partielle et émiettée a pour conséquence de multiplier les circuits proposés, rendant la pratique peu lisible pour les compétiteurs et le public, et peu visible médiatiquement.

Le modèle fédéral sportif ne parait pas, a priori, être approprié au milieu du sport électronique et cela, semble-t-il, principalement pour trois raisons. Tout d'abord, contrairement aux jeux sportifs qui sont des pratiques autonomes, les jeux vidéo sont la création d'éditeurs, c'est-à-dire de sociétés qui prennent en charge le financement, parfois le développement, et surtout la commercialisation des jeux, afin d'en assurer la diffusion. Il n'existe pas de tournois de jeux de tir, de jeux de combat ou de jeux de stratégie, mais bien des compétitions de Counter Strike, de Street Fighter ou de Starcraft. Les associations doivent donc effectuer des partenariats avec ces sociétés privées pour avoir le droit d'organiser des événements sur les jeux qu'ils promeuvent, ce que toutes n'acceptent pas systématiquement. Ensuite, le jeu vidéo s'est développé au cours des dernières décennies indépendamment de toute instance centralisatrice, laissant aux pratiquants la possibilité de se structurer et de s'organiser de manière originale et inédite. C'est ainsi que les joueurs ont préféré adopter dans un premier temps le système autogéré de "clans " et de "guildes ", à celui de clubs sportifs, les libérant de toute contrainte institutionnelle. Lorsque certaines instances fédératrices ont voulu investir le champ du jeu vidéo compétitif, elles se sont exposées à la réticence de joueurs contestant leur légitimité. Enfin, les plus puissantes institutions sportives nationales et reconnues comme telles (le ministère de la Ville, de la Jeunesse et des Sports et les fédérations sportives nationales en tête), n'ont jamais prêté attention à ce phénomène. Ce désintérêt du mouvement sportif pour le jeu vidéo compétitif semble prendre racine dans le flou sémantique du terme "sport » et la présupposée opposition entre activités physiques et jeu vidéo évoquée dans la première partie de cet article. Ces institutions définissent encore majoritairement le sport par les critères de l'effort physique et de la dépense énergétique et imaginent encore difficilement comment promouvoir la pratique du jeu vidéo auprès de la population française au même titre que l'athlétisme, le tennis ou le rugby. Le supposé caractère enfantin, désocialisant ou parfois violent du jeu vidéo, favorisant un peu plus la stigmatisation de la pratique et de ses pratiquants, semble avoir pour un temps, détourné l'institution sportive du sport électronique.

Contrairement à la Corée du Sud, dont le gouvernement promeut activement le sport électronique par l'intermédiaire de la Korean esport Player Association (KeSPA), sous l'égide du Ministère de la Culture, des Sports et du Tourisme, la France reste, comme de nombreux autres pays européens, hermétique à la légitimation fédérale du jeu vidéo compétitif. L'organisation sud-coréenne, fondée en 2000 , fait ainsi office de modèle à atteindre pour tous les acteurs du sport électronique des autres pays du monde. Elle supervise un peu plus de vingt jeux pratiqués sous forme compétitive, en régissant leurs compétitions, leurs règlementations et leur diffusion au travers des différents médias. ${ }^{14}$ 


\section{Conclusion : le jeu vidéo compétitif, un « sport parallèle »?}

Conjointement à l'institutionnalisation associative publique française du jeu vidéo compétitif, coexiste un second modèle de structuration, cette fois-ci professionnel et international, bien plus spectaculaire et médiatisé que le premier. Malgré sa relative jeunesse, le sport électronique se professionnalise très rapidement, année après année, et se développe selon un modèle original, indépendamment de toute influence fédérale en tant que dispositif organisationnel. En effet, il s'est affranchi du modèle pyramidal des fédérations sportives, soit en déléguant l'organisation des manifestations à des organismes privés spécialisés dans l'événementiel vidéoludique et/ou le divertissement audiovisuel (Oxent pour l'Electronic Sports World Cup, GOM eXP pour la Global Starcraft II League, Modern Times Group pour l'Electronic Sports League ou Activision pour la Major League Gaming), soit en laissant l'éditeur du jeu organiser lui-même son championnat et les événements associés (comme les Starcraft II World Championship Series organisées par Blizzard Entertainment ou le tournoi The International sur DoTA 2 supervisé par l'éditeur Valve).

Ce modèle organisationnel semble faire écho au concept de "para-sport » récemment développé par Bordes (2015), qui désigne «une pratique physique compétitive, réglementée par un code de jeu relevant d'une instance supérieure autonome, déconnectée des organisations sportives reconnues comme légitimes». C'est le cas notamment des $X$-Games (compétition internationale annuelle de "sports de glisse ») organisés et diffusés par le groupe médiatique américain ESPN, ou bien du circuit mondial de plongeon de falaise (Cliff Diving) organisé par l'entreprise autrichienne Red Bull. Ainsi les grands événements e-sportifs que sont la Major League Gaming (MLG) ou l'Evolution Championship Series (EVO) en Amérique du Nord, l'Electronic Sports League (ESL) et la Dreamhack (DH) en Europe, ou l'Electronic Sports World Cup (ESWC) à travers le monde, sont opérés et financés par des sociétés et des investisseurs privés. Ces sociétés décident des modalités de déroulement des épreuves, de la règlementation, de l'arbitrage et donc des sanctions, des récompenses, et des caractéristiques (âge minimum, statut professionnel) des participants. Les joueurs professionnels (pro-gamers) sont alors recrutés par des structures sponsorisées par des investisseurs à la logique explicitement commerciale, et financés pour participer aux compétitions en leur nom, créant de ce fait une rupture nette entre l'amateurisme et le professionnalisme (voir Figure 4). 


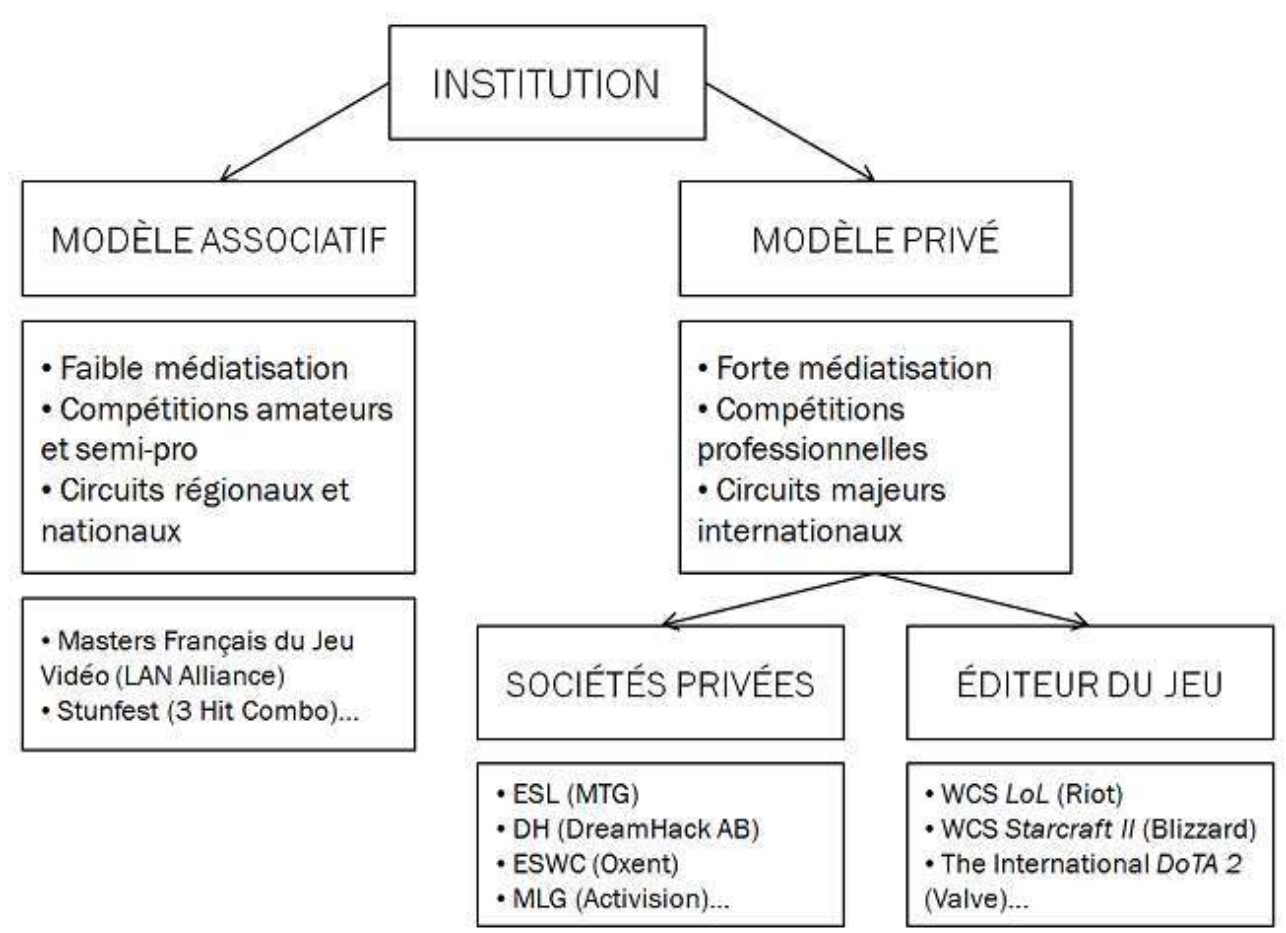

31

Le cas du jeu League of Legends offre quant à lui un éclairage singulier sur ce que pourrait être l'e-sport et les para-sports de demain. La structuration de son système compétitif et règlementaire est instituée par son développeur et éditeur Riot Games, l'inscrivant de ce fait dans le modèle parallèle du sport moderne décrit ci-dessus. Mais, depuis août 2012 et la création des Challenger Series, il propose une nouvelle variation de ce modèle, rapprochant finalement un peu plus le dispositif organisationnel du sport électronique de celui du sport moderne. Les Challenger Series ont été mises en place par Riot Games pour créer une continuité directe entre l'amateurisme et le professionnalisme, réinstaurant le principe méritocratique et d'ascension pyramidale du sport moderne. Chaque joueur peut ainsi clairement identifier le chemin qu'il lui faut parcourir pour prétendre et accéder aux divisions professionnelles. À l'instar du sport moderne qui, selon son modèle, peut permettre à un pratiquant de gravir une succession de divisions, du championnat départemental au championnat régional, puis national et enfin international, une équipe de League of Legends peut espérer un jour accéder, au fil des étapes, aux Championship Series (LCS), puis aux World Championships (WCS), pour lesquels elle sera directement rémunérée par Riot Games. Le jeu League of Legends fait donc office de précurseur dans le monde de l'e-sport, érigeant le premier un pont évident entre le monde amateur et le monde professionnel.

$\mathrm{Au}$ regard du succès phénoménal qu'il rencontre depuis sa mise en place, ce cas particulier pourrait bien être la norme d'ici quelques années. L'organisation du modèle instauré par Riot Games professionnalise ainsi d'une part les joueurs en leur assurant une rémunération régulière, et d'autre part la pratique, offrant une meilleure lisibilité de son fonctionnement envers le public. Les revenus financiers générés par le jeu permettent à Riot Games de proposer des manifestations e-sportives de plus en plus spectaculaires chaque année, favorisant ainsi sa popularisation à travers le monde. Les phases finales 
des World Championships 2014 qui se sont tenues dans le stade Sang-am de Séoul, célèbre pour avoir été l'une des principales enceintes sportives de la Coupe du Monde de football FIFA 2002, ont ainsi réuni 40000 spectateurs venus encourager leur équipe favorite lors d'un show digne du Super Bowl américain. Pour l'édition suivante, dont la finale entre les deux équipes sud-coréennes SKT et KOO Tigers se tenait le 31 octobre 2015 à la MercedesBenz Arena de Berlin devant près de 17000 personnes, Riot Games a alors enregistré 36 millions de spectateurs uniques à travers le monde lors de l'événement, avec un pic à 14 millions simultanément ${ }^{15}$.

À titre de comparaison, le seul match décisif des finales NBA (National Basket Association) de la même année opposant les Cleveland Cavaliers au Golden State Warriors s'est tenu dans un stade d'un peu plus de 20000 personnes, et a été suivi par un peu plus de 23 millions de téléspectateurs simultanément rien qu'aux États-Unis ${ }^{16}$. Bien qu'encore en deçà de celles du sport moderne (et notamment des géants médiatiques que sont la Coupe du monde de football ou les Jeux Olympiques d'été), ces audiences en constante progression assurent, dans une certaine mesure, le financement des compétitions et permettent ainsi à Riot Games de récompenser l'équipe victorieuse d'une somme d'un million de dollars (environ 910000 euros), lorsque les organisateurs de Roland Garros dotent les vainqueurs individuels de leur tournoi d'un peu plus d'un million et demi d'euros. ${ }^{17}$

En associant la spécificité de l'institutionnalisation privée, propre aux para-sports, au format pyramidal classique d'accession au haut-niveau du sport moderne, le jeu League of Legends pourrait représenter une forme aboutie du dispositif organisationnel $\mathrm{du}$ sport électronique de demain. C'est, tout au moins, ce que laisse présager le succès grandissant des trois dernières saisons de compétitions.

\section{BIBLIOGRAPHIE}

ADAMUS T. (2012), « Playing computer games as electronic sport: In search of a theoretical framing for a new research field », In Fromme J. \& Unger A. (eds.), Computer Games and New Media Cultures: A Handbook of Digital Game Studies, Berlin: Springer, pp. 477-490.

BESOMBES N. (2015), « Du streaming au mainstreaming : mécanismes de médiatisation du sport électronique », In Obœuf A. (dir.), Sport et médias, Paris, éditions du CNRS, pp.179-189.

BORDES P. (2015), « Médias et para-sports. La fabrique de l'extrême », In Obœuf A. (dir.), Sport et médias, Paris, éditions du CNRS, pp. 169-177.

BOUTET M. (2003), « Des jeux d'adultes ? Corporéités et sociabilités dans les cyberespaces ludiques », In Roustan M. (dir.), La pratique du jeu vidéo : réalité ou virtualité ?, Paris, L'Harmattan, pp. 99-113.

CALLÈDE J.-P. (1993), Histoire du sport en France. Du Stade Bordelais au S.B.U.C. (1889-1939), Talence, éditions de la M.S.H.A. 
CLAIS J.-B. et ROUSTAN M. (2003), « Le jeu vidéo, c'est physique ! Réalité virtuelle et engagement du corps dans la pratique vidéoludique ", In Roustan M. (dir.), La pratique du jeu vidéo : réalité ou virtualité ?, Paris, L'Harmattan, 2003, pp. 35-52.

DE COUBERTIN P. (1972), Pédagogie sportive. Histoire, technique, action morale et sociale des exercices sportifs, Paris, Vrin.

DEFRANCE J. (1995), Sociologie du sport, Paris, La Découverte.

ELIAS N. \& DUNNING É. (1986), Sport et civilisation. La violence maîtrisée, [1994], Paris, Fayard. GUTTMANN A. (1978), From Ritual to Record. The Nature of Modern Sports, New York, Columbia University Press.

HÉAS S. \& MORA P. (2003), « Du joueur de jeux vidéo à l'e-sportif : vers un professionnalisme florissant de l'élite? », In Roustan M. (dir.), La pratique du jeu vidéo : réalité ou virtualité ? Paris, L'Harmattan, pp. 131-146.

JONASSON K. \& THIBORG J. (2010), « Electronic sport and its impact on future sport », Sport in Society, vol. 13, n², pp. 287-299.

MAGNANE G. (1962), Sociologie du sport. Situation du loisir sportif dans la culture contemporaine, Paris, Gallimard.

MAUSS M. (1950), Sociologie et anthropologie, Paris, PUF.

MORA P. (2005), « Derrière l'e-sport : un conflit d'experts de jeux réseaux compétitifs », In Fortin T. et al, Les jeux vidéo : pratiques contenus et enjeux sociaux, Paris, L'Harmattan, pp. 25-122.

MORA P. (2009), « Des J.O à la FFJV : PES et la construction sociale du sport », Les cahiers du jeu vidéo, $\mathrm{n}^{\circ}$ 2, Football Stories, pix'n love, pp. 140-150.

PABERZ C. (2012), « Le jeu vidéo comme sport en Corée du Sud ? ", Hermès, La revue, n62, Paris, éditions du CNRS, pp. 48-51.

PARLEBAS P. (1986), Éléments de sociologie du sport, Paris, PUF.

PARLEBAS P. (1999), Jeux, sports et sociétés, Lexique de praxéologie motrice, Paris, INSEP-Publications.

PETER C. (2007), « Goût pour les jeux vidéo, goût pour le sport, deux activités liées chez les adolescents $"$, Culture prospective, $\mathrm{n}^{\circ} 2$.

POCIELLO C. et al. (1981), Sports et Société. Approche socio-culturelle des pratiques, Paris, Vigot.

ROUSTAN M. (dir.) (2003), La pratique du jeu vidéo : réalité ou virtualité ?, coll. « Dossiers Sciences Humaines et Sociales », série «Consommations \& Sociétés », Paris, L'Harmattan.

ROUSTAN M. (2004), « La pratique du jeu vidéo : expériences de réalité virtuelle ? », Champs culturels, $\mathrm{n}^{\circ} 18$ « Virtualité, art et culture », p. 16-19.

TAYLOR T. L. \& WITKOWSKI E. (2010), « This is how we play it: What a mega-LAN can teach us about games ", In Pisan Y. (ed), Proceedings of the Fifth International Conference on the Foundations of Digital Game, Monterey, CA: ACM Press.

TAYLOR T. L. (2012), Raising the Stakes: E-Sports and the Professionalization of Computer Gaming, Cambridge, MIT Press.

TERRET T. (2007), Histoire du sport, Paris, PUF.

TRÉMEL L. (2001), « Les « jeux vidéo » : un ensemble à déconstruire, des pratiques à analyser », Revue française de pédagogie, $\mathrm{n}^{\circ} 136$. 
TRICLOT M. (2011), Philosophie des jeux vidéo, Paris, Zones.

ULMANN J. (1977), De la gymnastique aux sports modernes. Histoire des doctrines de l'éducation physique , Paris, Vrin.

WAGNER M. G. (2006), « On the scientific relevance of eSports », Proceedings of the 2006 International Conference on Internet Computing and Conference on Computer Game Development, Las Vegas, CSREA Press, pp. 437-440.

WARNIER J.-P. (1999), Construire la culture matérielle. L'homme qui pensait avec ses doigts, Paris, PUF. WITKOWSKI E. (2009), « Probing the sportiness of eSports », In Christophers J. \& Scholz T. (eds.), esports yearbook 2009, Norderstedt, Germany: Books on Demand GmbH, pp. 53-56.

WITKOWSKI E. (2012), « On the Digital Playing Field: How We "Do Sport" With Networked Computer Games », Games and Culture, vol. 7, n 5, pp. 349-374.

\section{NOTES}

1. Source : eSports: Digital Games Brief, April 2014. SuperData Research. www.superdataresearch.com.

2. Source : eSports Market Brief, October 2015. SuperData Research. www.superdataresearch.com.

3. La consultation publique lancée par le gouvernement entre le 26 septembre et le 18 octobre 2015 dans le cadre de la loi «République Numérique » appelait la population française à donner son avis sur une première mouture du texte qui légiférera une grande partie des nouvelles activités liées à Internet. Les internautes ont ainsi pu exprimer leur souhait de voir l'e-sport s'éloigner des considérations de jeux d'argent en ligne pour se rapprocher des pratiques sportives. Consultable en ligne à l'adresse https://www.republique-numerique.fr/.

4. «My modest hope is that this book provides an early contribution [...] to what will hopefully be an ongoing collection of research, including perhaps not only game studies, but fields like leisure studies or even the sociology of sport » (2012, p. 30).

5. Un trait pertinent en linguistique est un élément qui remplit une fonction distinctive. La pertinence motrice permet donc de distinguer le jeu sportif du jeu non-sportif. Cette notion oppose les jeux dont la réalisation de la tâche nécessite une maîtrise corporelle, c'est-à-dire une réussite motrice (comme dans les osselets ou le mikado), des jeux où priment le hasard (les jeux de dés ou de cartes), la combinatoire (les échecs) ou la fiction et l'imaginaire (les jeux de déguisements et de simulacre).

6. Source: http://www.riotgames.com/articles/20140711/1322/league-players-reach-newheights-2014.

7. Jean-Pierre Warnier définit la synthèse corporelle (ou schéma corporel) comme « la perception synthétique et dynamique qu'un sujet a de lui-même, de ses conduites motrices, et de sa position dans l'espace-temps. Elle mobilise l'ensemble des sens dans leur rapport au corps propre et à la culture matérielle. [Elle] est le résultat d'apprentissages qui se poursuivent et s'entretiennent au cours de l'existence entière. Elle fait preuve d'une grande variabilité individuelle et sociale [...]. Elle se dilate et se rétracte tour à tour pour intégrer de multiples objets [...] dans les conduites motrices du sujet. » (1999, p. 27).

8. Pierre Parlebas définit l'inconscient moteur comme : «le contenu et l'organisation des conduites motrices d'une personne agissante traduisant le fait qu'une très faible partie seulement des opérations déclenchées [...] fait l'objet d'une réelle "prise de conscience" » (1999, p. 170).

9. Source : http://thecpl.com/about-cpl/. 
10. Source : http://www.eswc.com/fr/page/about.

11. Source : esportsearnings.com.

12. Source : 2015 World Championship Rules, Version 1.01, 7 juillet 2015.

13. Le 27 octobre 2015, à l'occasion de la Paris Games Week, s'est tenue une journée de débat et de réflexions intitulée esport Summit, au cours de laquelle une série de présentations et tables rondes animées par un panel d'entrepreneurs et d'observateurs de l'industrie du jeu vidéo compétitif visaient à « Comprendre l'industrie des compétitions de jeu vidéo ». À la suite de cet échange, les différents acteurs représentatifs du milieu e-sportif présents ont cosigné un compterendu destiné à Axelle Lemaire, secrétaire d'État au numérique. S'en est ensuivi moins d'un mois après, une rencontre entre certains des cosignataires et des représentants du ministère de la Ville, de la Jeunesse et des Sports, en vue d'offrir un cadre législatif aux joueurs professionnels et à l'e-sport.

14. Source : http://wiki.teamliquid.net/starcraft2/Korea_e-Sports_Association.

15. Source : leagueoflegends.com, "Worlds 2015 Viewership"

16. Source : forbes.com, "Inside the Numbers : 2015 NBA Finals were highest rated ever for ABC"

17. Source : rolandgarros.com, "Prize Money"

\section{RÉSUMÉS}

La comparaison entre jeux vidéo compétitifs et jeux sportifs fait régulièrement débat, et ce notamment auprès des acteurs de la communauté du sport électronique. À l'aide de concepts empruntés à la sociologie du sport, l'article propose de questionner la définition du sport électronique au regard de quatre critères objectifs et opérationnels régulièrement utilisés pour définir le jeu sportif: la pertinence motrice de l'activité, l'organisation de compétitions, le système règlementaire et le dispositif institutionnel. Cette analyse a pour objectif d'apporter un éclairage original sur le phénomène du jeu vidéo compétitif et sur le processus de sportification qui le traverse, à travers l'exemple du jeu vidéo League of Legends.

The comparison between competitive video games and modern sports is regularly subject of debate and especially among the electronic sport community. Using concepts borrowed from the sociology of sport, the paper questions the definition of e-sport through four objective and operational criteria used to define modern sports: the motor skills, the organization of competitions, the regulatory system, and the institutional governing bodies. This analysis, which mainly takes support on the competitive video game League of Legends, aims to enlighten the sportisation process of competitive video gaming.

\section{INDEX}

Mots-clés : sport électronique, jeu vidéo compétitif, sport, conduite motrice, compétition, règlement, institution

Keywords : electronic sport, competitive video game, modern sport, motor skill, competition, rules, institution 


\section{AUTEUR}

\section{NICOLAS BESOMBES}

Université Paris Descartes 